\title{
Indirect Ultrasound Guidance Increased Accuracy of the Glenohumeral Injection Using the Superior Approach: A Cadaveric Study of Injection Accuracy
}

\author{
Joon Sung Kim, MD, Min-Wook Kim, MD, Dong Yoon Park, MD
}

Department of Physical Medicine and Rehabilitation, St. Vincent's Hospital, The Catholic University of Korea College of Medicine, Suwon, Korea

Objective To investigate whether or not indirect ultrasound guidance could increase the accuracy of the glenohumeral joint injection using the superior approach.

Methods Twelve shoulders from 7 adult cadavers were anatomically dissected after a dye injection had been performed, while the cadavers were in the supine position. Before the injection, a clinician determined the injection point using the ultrasound and the more internal axial arm rotation was compared to how it was positioned in a previous study. Injection confidence scores and injection accuracy scores were rated.

Results The clinician's confidence score was high in $92 \%$ ( 11 of 12 shoulders) and the injection accuracy scores were $100 \%$ ( 12 of 12 shoulders). The long heads of the biceps tendons were not penetrated.

Conclusion Indirect ultrasound guidance and positioning shoulder adducted at $10^{\circ}$ and internally rotated at $60^{\circ}-$ $70^{\circ}$ during the superior glenohumeral joint injection would be an effective method to avoid damage to the long head of biceps tendons and to produce a highly accurate injection.

Keywords Shoulder, Injections, Ultrasound, Superior, Cadaver

\section{INTRODUCTION}

Injections are frequently made to the glenohumeral

Received May 4, 2012; Accepted September 13, 2012

Corresponding author: Dong Yoon Park

Department of Physical Medicine and Rehabilitation, St. Vincent's Hospital, The Catholic University of Korea College of Medicine, 93 Jungbu-daero, Paldal-gu, Suwon 442-723, Korea

Tel: +82-31-249-7650, Fax: +82-31-251-4481, E-mail: dyfree@naver.com

(c) This is an open-access article distributed under the terms of the Creative Commons Attribution Non-Commercial License (http://creativecommons. org/licenses/by-nc/3.0) which permits unrestricted noncommercial use, distribution, and reproduction in any medium, provided the original work is properly cited.

Copyright $\odot 2013$ by Korean Academy of Rehabilitation Medicine joint as part of treatment for adhesive capsulitis. We reported high accuracy of glenohumeral joint injections using a superior approach based on a previous study. However, that approach risked penetrating to the long head of the biceps brachii tendon [1].

Recently, ultrasound has been used to increase the accuracy of the needle placement of intra-articular injections. There are 2 methods to approach the needle target, the indirect technique and the direct technique. In the indirect technique, an ultrasound is used to confirm the puncture site and the location of the target; however this does not serve as a guide to advance the needle. Using the direct technique, the needle advancement and target 
are seen in real time under direct ultrasound control. The long head of the biceps tendon can be traced by using the ultrasound from the proximal site to just distal to the subacromial space.

The long head of the biceps tendon is affected dependent upon its location by rotating the arm externally or internally. The adducted and internally rotated arm position would be expected to prevent injury of that tendon from the needle tip during glenohumeral joint injection. The purpose of this study is to prove that adducted and internally rotated shoulder position would improve accuracy of injection and prevent biceps tendon injury during

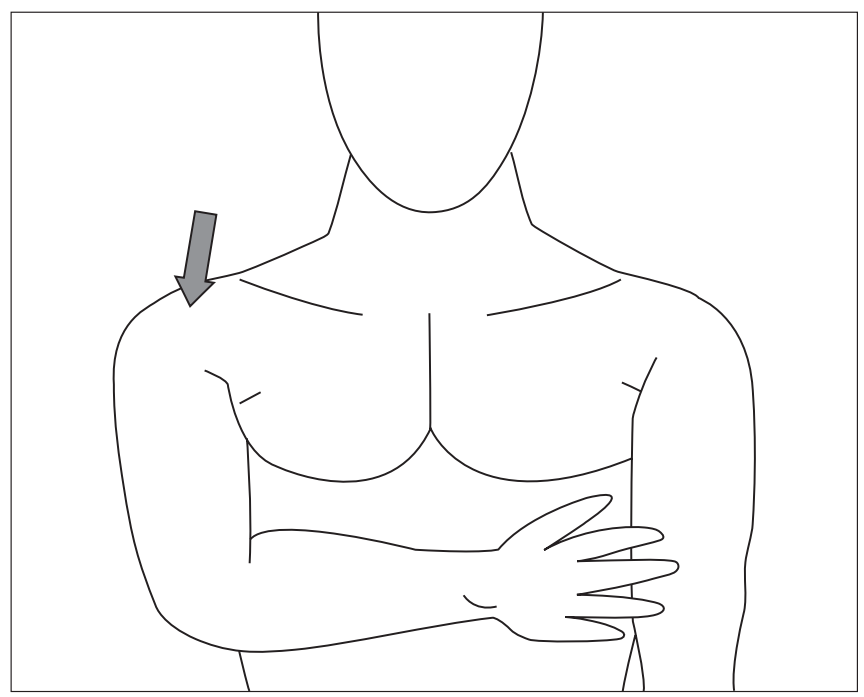

Fig. 1. The cadaver's hand is located on his/her abdomen. In this position, the elbow was flexed $90^{\circ}$, the shoulder was positioned at $10^{\circ}$ of adduction and at $60^{\circ}-70^{\circ}$ of internal rotation. The arrow indicates the injection on point. indirect, ultrasound-guided glenohumeral joint injection.

\section{MATERIALS AND METHODS}

Included in this study were 12 shoulders from 7 cadavers, whose relatives consented to their use for research purposes. Six of the shoulders used were from 4 formalinfixed cadavers and the other shoulders were from fresh cadavers.

The cadavers were supine, and their elbows were flexed $90^{\circ}$ and shoulders were positioned at $10^{\circ}$ adduction and at $60^{\circ}-70^{\circ}$ internal rotation, so that their hands were located on their abdomen (Fig. 1). This position is selected so that the needle does not damage the long head tendon of the biceps brachii. Before the injection was performed, ultrasonographic examination (HD11 XE ultrasound system; Philips Healthcare, Eindhoven, The Netherlands) of the shoulder was performed to identify the location of biceps brachii tendon by the clinician who was to perform the injection. The tendon had been traced from the distal aspect of bicipital groove to the proximal side by keeping the probe perpendicular to the axis of biceps tendon (Fig. 2). One $\mathrm{mL}$ of dye (blue ink) was injected into the glenohumeral joint using 1.5-inch 21-gauge needle. One experienced clinician performed the injection procedure. After the acromioclavicular joint was palpated, the site 5 $\mathrm{mm}$ lateral to just anterior to the aspect of the acromioclavicular joint was selected as the needle entry point. After the needle was inserted through the entry point, it was advanced in a $20^{\circ}$ lateral and a $20^{\circ}$ dorsal direction. When the resistance was felt at the tip of needle, we applied a gentle passive internal and external rotation to
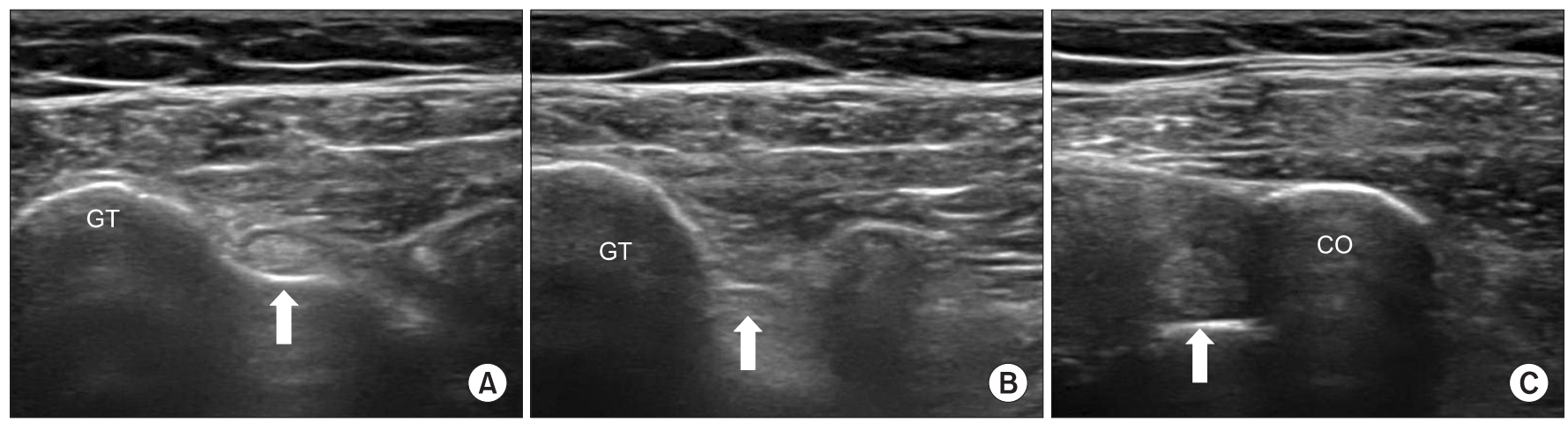

Fig. 2. In the transverse view of ultrasonography, the long head of biceps tendon (arrow) is seen in the bicipital groove level (A) and the more proximal level (B). The tendon adheres to the superior margin of the glenoid and is located beside the $\mathrm{CO}$ at the coracoid process level (C). GT, greater tubercle of humeral head; $\mathrm{CO}$, coracoid process. 
the shoulder. If the tip of the needle showed concurred movement to this motion, we pulled the needle slightly back, and then injected the dye.

A confidence score was rated according to clinician's opinion (Table 1). After the scoring, the tissues were carefully dissected to access to the joint to where the dye could be found. The needle and shoulder position were maintained during dissection to prevent needle displacement. Following the dissection, placement of the tip of the needle and dye were observed to determine whether the injected material had reached the intra-articular space and the long head of biceps brachii tendon has injured by the needle. Then, an injection accuracy score was determined (Table 2).
Table 1. Confidence score

\begin{tabular}{ll}
\hline Confidence (score) & \multicolumn{1}{c}{ Criteria } \\
\hline Inaccurate (1) & $\begin{array}{c}\text { Perception of hardness of the bi- } \\
\text { ceps tendon during injection after } \\
\text { needle retraction }\end{array}$ \\
& $\begin{array}{l}\text { Perception of high resistance during } \\
\text { injection after needle retraction } \\
\text { Observation of skin swelling after } \\
\text { injection }\end{array}$ \\
& $\begin{array}{l}\text { Clinician failed to feel the needle tip } \\
\text { touch the humeral head }\end{array}$ \\
Unclear (2) & $\begin{array}{l}\text { Unclear } \\
\text { Accurate (3) }\end{array}$ \\
\hline
\end{tabular}

Table 2. Accuracy score

\begin{tabular}{cl}
\hline Degree & \multicolumn{1}{c}{ Accuracy } \\
\hline 1 & Miss \\
2 & Correct and another site \\
3 & Correct site only \\
\hline
\end{tabular}

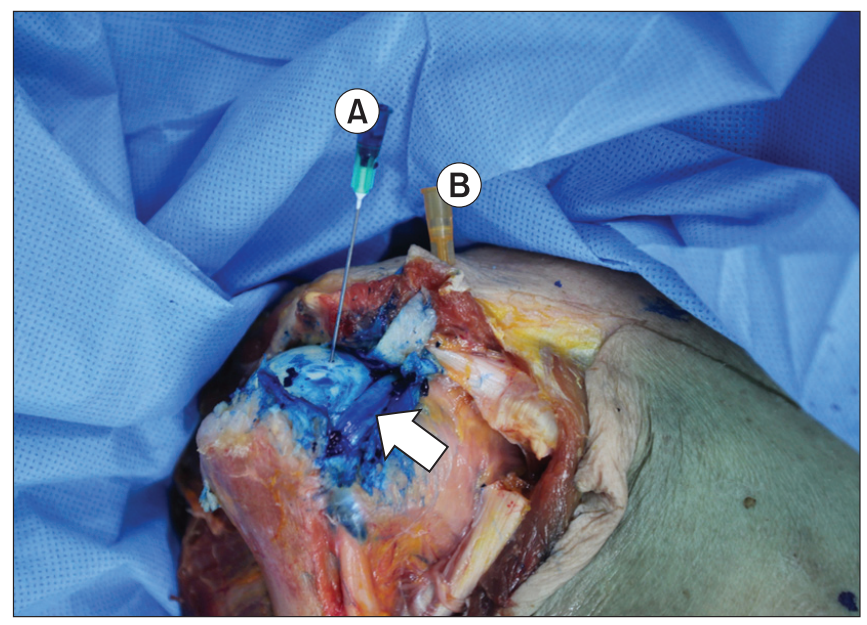

Fig. 3. Blue dye is seen in the glenohumeral joint cavity. The blue needle (A) was inserted through the anterolateral side of the acromioclavicular joint and is located on the lateral side of the long head of biceps brachii tendon (arrow). The yellow needle (B) is the landmark of the anterior acromioclavicular joint.

Table 3. Summary of twelve shoulders

\begin{tabular}{ccccccccccc}
\hline $\begin{array}{c}\text { Cadaver Shoulder } \\
\text { no. }\end{array}$ & no. & Sex & $\begin{array}{c}\text { Age } \\
\text { (yr) }\end{array}$ & Side & $\begin{array}{c}\text { Fixed } \\
\text { type }\end{array}$ & $\begin{array}{c}\text { Confidence } \\
\text { score }\end{array}$ & $\begin{array}{c}\text { Dye in } \\
\text { GH space }\end{array}$ & $\begin{array}{c}\text { Dye in } \\
\text { SASD bursa }\end{array}$ & $\begin{array}{c}\text { Biceps tendon } \\
\text { injury }\end{array}$ & $\begin{array}{c}\text { Accuracy } \\
\text { score }\end{array}$ \\
\hline 1 & 1 & F & 93 & R & Formalin & 3 & Present & Absent & No & 3 \\
& 2 & & & L & Formalin & 3 & Present & Present & No & 2 (SASD bursa) \\
\hline 2 & 3 & M & 77 & L & Formalin & 3 & Present & Present & No & 2 (SASD bursa) \\
3 & 4 & F & 78 & L & Formalin & 3 & Present & Absent & No & 3 \\
\hline 4 & 5 & M & 69 & R & Formalin & 3 & Present & Absent & No & 3 \\
& 6 & & & L & Formalin & 3 & Present & Absent & No & 3 \\
\hline 5 & 7 & M & 74 & R & Fresh & 3 & Present & Absent & No & 3 \\
& 8 & & & L & Fresh & 1 & Present & Absent & No & 3 \\
\hline & 9 & F & 77 & R & Fresh & 3 & Present & Absent & No & 3 \\
& 10 & & & L & Fresh & 3 & Present & Absent & No & 3 \\
\hline & 11 & M & 80 & R & Fresh & 3 & Present & Absent & No & 3 \\
\hline
\end{tabular}

R, right; L, left; GH, glenohumeral; SASD, subacromial-subdeltoid. 


\section{RESULTS}

The average age of the cadavers in life was 78 years (range, 69 to 93 years). Three of the cadavers were female and the other 4 were male.

The detailed results are shown in Table 3. Blue dye was found at the glenohumeral joint spaces in all 12 shoulders (Fig. 3). The accuracy of glenohumeral injection using superior approach with internally rotated shoulder was $100 \%$ (12 of 12 shoulders). However, blue dye was found at the subacromial-subdeltoid bursa in 2 of the 12 shoulders. The clinician's confidence scores were high in $92 \%$ (11 of 12 shoulders). In all cases, the needle passed lateral side to the long head tendon of the biceps brachii and did not penetrate that tendon.

\section{DISCUSSION}

When an indirect ultrasound-guided technique was used, the accuracy of injection increased to $100 \%$ and injury to the long head of bicep brachii tendon did not occur $(0 \%)$. There are 4 important differences between previous studies that used the superior approach and this study [1]. First, the ultrasound was used for identifying the location of the long head of biceps brachii tendon before needle insertion in this study. Ultrasonography was used so that the safety of this area could be confirmed in a clinical setting. Second, the shoulder position of cadaver was changed. The shoulder had been positioned at $10^{\circ}$ internal rotation with no adduction in previous study. In this study, the position was $10^{\circ}$ adduction and $60^{\circ}-70^{\circ}$ internal rotation, so that the angle of the axial rotation could increase internally. Third, the needle entry point was moved from anterior aspect of acromioclavicular joint in a previous study to $5 \mathrm{~mm}$ laterally in the current study. Finally, the needle advancement in a previous study, which had been a $10^{\circ}$ lateral and a $10^{\circ}$ dorsal direction, was changed to $20^{\circ}$ lateral and $20^{\circ}$ dorsal direction. The needle was more dorsolaterally tilted to access the top of the glenohumeral joint capsule.

Despite the high accuracy of the superior approach, needle injury to long head of the biceps brachii tendon occurred in $15.8 \%$ in previous cadaver studies $[1,2]$. The greater the shoulder is internally rotated, the more the long head tendon of biceps brachii moves toward the medial side [3]. The synergic effect of more laterally located needle from $10^{\circ}$ to $20^{\circ}$ and to medially locate the long head tendon of biceps brachii was expected to prevent injury of that tendon from the needle. As we expected, no damage to long head tendon of biceps brachii occurred in this study ( $0 \%)$.

Recently, it has been reported that the direct ultrasound-guided method increased the accuracy of glenohumeral joint injection. A relatively high accuracy was achieved by that method. Rutten et al. [4] reported a $96 \%$ success rate with anterior approach and $92 \%$ with posterior approach. Needle tip and target could be visualized in a real time with direct ultrasound-guided injection technique. However, this technique may increase chance of infection because ultrasound transducer or gel could be a medium for the microorganism contamination, such as staphylococcal infection [5]. Thus, it requires aseptic gel and sterile envelopes for the probe in order to minimize the risk of infection. It cost more money than the indirect method to use these instruments. The clinician who performs this technique should coordinate between the needle insertion and handling of the probe. It is somewhat more difficult for the non-experienced person to perform. In addition, the needle should advance inline and parallel to the ultrasound transducer to improve visualization of the needle tip in the direct technique. This increases the distance from the needle entry point at skin to the target. The shorter distance could be achieved in the indirect technique than the direct technique. If a high accuracy around $100 \%$ could be achieved by the indirect technique resolving these disadvantages of direct technique, indirect technique seems more useful in clinical settings.

In addition to these advantages with indirect ultrasound-guided technique, we used a superior approach method. The superior approach has many advantages [6]. This approach has the relatively shorter distance from skin to glenohumeral joint space than anterior or posterior approaches. It is easy to palpate acromioclavicular joint, which is used for landmark of needle entry point. Finally, in this approach, there is no major nerve or vessels on the course of needle compared with anterior or posterior approaches. The anterior approach could damage the cephalic vein, axillary artery, and the brachial plexus. Posterior approach would damage the supraclavicular nerve and circumflex scapular vessels $[7,8]$.

The supraspinatus muscle is penetrated during the su- 
perior approach. However, it is expected that there are no significant harms. The penetrated muscle location is far from the supraspinatus tendon, so risk of the tendon injury is very low. Injury to the supraspinatus muscle is limited because the muscle is frequently penetrated during arthroscopy via superior portal and the needle used in shoulder injection and has much a smaller diameter than the scope used in arthroscopy [9].

Considering glenohumeral joint has been mostly injected to patients with adhesive capsulitis, the internally rotated shoulder position during procedure would be expected to be easier to perform. Because most of the patients with adhesive capsulitis show limitation of active and passive range of motion mainly affects external rotation and abduction than internal rotation [10].

Blue dye was found at subacromial-subdeltoid bursa space in $16.7 \%$ ( 2 of 12 shoulders). In those 2 cadavers, blue dye was also found at the glenohumeral joint space at the same time. This is explained by possibility of full thickness rotator cuff tear. The prevalence of rotator cuff tears in asymptomatic patient was increased with age of patients. Tempelhof et al. [11] reported that the prevalence of rotator cuff was $31 \%$ in patient aged 70 to 79 years and $51 \%$ in aged over 81 years. The ages of the 2 cadavers at which shoulders blue dye was found were 77 and 93 years. Generally glenohumeral joint space and subacromial-subdeltoid bursa space are separated, but in a rotator cuff tear, those spaces are connected to each other [8]. Therefore, blue dye could be seen at subacromial-subdeltoid bursa space in those cases despite of correct injection.

Although sitting position is preferred to supine during in outpatient clinical settings, it is difficult to simulate shoulder injection with cadaver with sitting position. In cadaver, subluxation of the shoulder joint occurred in this position because no dynamic forces exist against gravity. The cadavers with supine position have been involved as the subject to resolve these problems.

There are some limitations in this study. The clinician who performed injections in this study has had wide experience with the superior approach. The success rate of the superior approach could be different from the rate in this study depending on the experience of the clinician. The needle tip touched the articular surface of the humerus once, during whole procedure, it can damage the articular cartilage. However, it hasn't been reported yet whether touching of the needle tip to humeral head during injection procedure would cause damage to the cartilage. Furthermore, it would occur even if the direct ultrasound-guided technique or the fluoroscopic guided technique is done.

In conclusion, the combination of indirect ultrasoundguided technique and positioning the shoulder internally rotated at $60^{\circ}-70^{\circ}$ during the superior approach of glenohumeral joint injection would be very effective way to avoid damage to the long head tendon of the biceps brachii and to produce a highly accurate injection.

\section{CONFLICT OF INTEREST}

No potential conflict of interest relevant to this article was reported.

\section{REFERENCES}

1. Kim JS, Yun JS, Kim JM, Ko YJ, Rhee WI, Ha NK, et al. Accuracy of the glenohumeral injection using the superior approach: a cadaveric study of injection accuracy. Am J Phys Med Rehabil 2010;89:755-8.

2. Johnson TS, Mesfin A, Farmer KW, McGuigan LA, Alamo IG, Jones LC, et al. Accuracy of intra-articular glenohumeral injections: the anterosuperior technique with arthroscopic documentation. Arthroscopy 2011;27:745-9.

3. Itoi E, Motzkin NE, Morrey BF, An KN. Stabilizing function of the long head of the biceps in the hanging arm position. J Shoulder Elbow Surg 1994;3:135-42.

4. Rutten MJ, Collins JM, Maresch BJ, Smeets JH, Janssen CM, Kiemeney LA, et al. Glenohumeral joint injection: a comparative study of ultrasound and fluoroscopically guided techniques before MR arthrography. Eur Radiol 2009; 19:722-30.

5. Ohara T, Itoh Y, Itoh K. Ultrasound instruments as possible vectors of staphylococcal infection. J Hosp Infect 1998;40:73-7.

6. Kim JS, Kwon JY, Jeong IS, Rhee WI, Im S, Kim HJ, et al. The success rate of superior approach glenohumeral injection in patients with frozen shoulder. J Korean Acad Rehabil Med 2007;31:37-40.

7. Hulstyn MJ, Fadale PD. Arthroscopic anatomy of the shoulder. Orthop Clin North Am 1995;26:597-612.

8. Stitik TP, Foye PM, Fossati J. Shoulder injections for 
osteoarthritis and other disorders. Phys Med Rehabil Clin N Am 2004;15:407-46.

9. Wolf EM. Anterior portals in shoulder arthroscopy. Arthroscopy 1989;5:201-8.

10. Wong PL, Tan HC. A review on frozen shoulder. Singa- pore Med J 2010;51:694-7.

11. Tempelhof S, Rupp S, Seil R. Age-related prevalence of rotator cuff tears in asymptomatic shoulders. J Shoulder Elbow Surg 1999;8:296-9. 